\title{
Diagnostic de l'incidence des Pathogènes Fongiques du Riz au Sud Bénin et Identification Morphologique de Cercospora oryzae
}

\author{
Soura B. Hervé, \\ Gnancadja L. S. André,
}

Université d'Abomey-Calavi, Département de Biologie Végétale, Laboratoire de Physiologie Végétale et Etude des Stress Environnementaux, Faculté des Sciences et Techniques, Benin (FAST/UAC)

Zandjanakou-Tachin Martine,

Université Nationale d'Agriculture, Laboratoire de Phytopathologie, Horticulture et Gestion des Espaces Verts, Bénin

\section{Koita Kadidia,}

Université de Ouagadougou I Prof Joseph Ki-Zerbo, Département de

Biologie Végétale, Laboratoire Biosciences Equipe de Recherche

Phytopathologie et Mycologie Tropicale, Unité de Formation et de

Recherche Sciences de la Vie et de la Terre, Burkina Faso (UFR/SVT)

Doi: 10.19044/esj.2019.v15n12p346 URL:http://dx.doi.org/10.19044/esj.2019.v15n12p346

\section{Résumé}

Le riz (Oryza sativa L.), est l'une des céréales la plus cultivée au Bénin. Même si le pays est à vocation agricole, l'autosuffisance alimentaire n'a jamais été atteinte, et l'importation du riz est toujours importante chaque année. Pour le riz, les maladies constituent le facteur entraînant la mauvaise qualité et la réduction des récoltes. C'est la raison qui nous amène à réaliser le suivi de la maladie de riz présent au Bénin. L'incidence de la maladie a été évalué par l'observation visuelle des symptômes au champ et l'identité des pathogènes a été confirmée par les tests au laboratoire. Pour cela un échantillonnage a été réalisé dans 6 départements (Mono, Couffo, Ouemé, Plateau, Atlantique, Zou), 9 communes (Zè, Abomey-Calavi, Comé, GrandPopo, Adjohoun, Pobè, Adja Ouéré, Dogbo, Ouinhi) et 12 sites (Awokpa, Docomey, Gative, Matekpo, Kodé-Ague, Gouti, Odja-Igbagi, Houeli-Gaba, Kpodji, Aizè, Zaloko, Zoungo) au Sud du Bénin. Une enquête a été réalisée durant l'échantillonnage pour récolter les informations qui peuvent nous aider à l'identification de l'agent pathogène. L'isolement a été réalisé en utilisant les milieux standards pour champignons (PDA, Farine de riz, Agar-Agar). Les isolats ont été ensuite identifiés par l'observation des colonies sur boite de Pétri, par des observations microscopique des spores et mycéliums. Les 
symptômes trouvés sont très variés et l'incidence de certains d'entre eux est faible sauf la Cercosporiose et la Pyriculariose sur feuilles. Dans les sites de Gative, Docomey, Kpodji, Matekpo, les symptômes trouvés de la Cercosporiose ne varient pas et l'incidence est proche de $100 \%$. L'isolement des microorganismes à partir d'échantillons de chacune de ces régions confirme les résultats obtenus dont Cercospora oryzae correspondait à l'espèce pathogène dominante dans le site de Gative, Docomey et Kpodji, Curvularia oryzae correspondait à l'espèce pathogène dominante dans le site de Aizè, Houeli Gaba, Zoungo, Magnaporthe grizaea correspondait à l'espèce pathogène dominante dans le site de Zaloko, Aizè, Docomey tandis que Helminthosporium oryzae correspondait à l'espèce pathogène dominante dans le site de Odja Igbagi, Awokpa, Kodé Ague. Ces différences trouvent probablement leur origine dans les conditions climatiques et le mode de culture différente entre les sites ainsi que dans l'utilisation des semences.

Mots-clés : Oryza sativa L., pathogène fongique, incidence, diagnostic, Identification morphologique 


\title{
Diagnosis of the Incidence of Fungal Pathogens of Rice in Southern Bénin, Morphological Identification of Cercospora Oryzae
}

\author{
Soura B. Hervé, \\ Gnancadja L. S. André,
}

Université d'Abomey-Calavi, Département de Biologie Végétale, Laboratoire de Physiologie Végétale et Etude des Stress Environnementaux, Faculté des Sciences et Techniques, Benin (FAST/UAC)

Zandjanakou-Tachin Martine,

Université Nationale d'Agriculture, Laboratoire de Phytopathologie, Horticulture et Gestion des Espaces Verts, Bénin

\section{Koita Kadidia,}

Université de Ouagadougou I Prof Joseph Ki-Zerbo, Département de

Biologie Végétale, Laboratoire Biosciences Equipe de Recherche

Phytopathologie et Mycologie Tropicale, Unité de Formation et de

Recherche Sciences de la Vie et de la Terre, Burkina Faso (UFR/SVT)

Abstract

Rice (Oryza sativa L.) is one of the most widely grown cereals in Benin. Even though the country is agricultural, food self-sufficiency has never been reached, and rice import is always important every year. For rice, diseases are the factor causing poor quality and the reduction of harvests. This is the reason that leads us to monitor the rice disease present in Benin. The incidence of the disease was assessed by visual observation of the symptoms in the field and the identity of the pathogens was confirmed by the laboratory tests. For this, a sampling was carried out in 6 departments (Mono, Couffo, Ouemé, Plateau, Atlantic, Zou), 9 municipalities (Zè, Abomey-Calavi, Comé, Grand-Popo, Adjohoun, Pobè, Adja Ouéré, Dogbo, Ouinhi) and 12 sites (Awokpa, Docomey, Gative, Matekpo, Kodé-Ague, Gouti, Odja-Igbagi, Houeli-Gaba, Kpodji, Aizè, Zaloko, Zoungo) in southern Benin. A survey was conducted during the sampling to gather information that can help us identify the pathogen. Isolation was carried out using standard mushroom media (PDA, Rice Flour, Agar-Agar). The isolates were then identified by observation of petri dish colonies by microscopic observations of spores and mycelia. The symptoms found are very varied and the incidence of some symptoms is low except cercosporiosis and rice blast on rice leaves. In the sites of Gative, 
Docomey, Kpodji, Matekpo, the found symptoms of cercosporiosis do not vary and the incidence is close to $100 \%$. The isolation of the microorganisms from samples of each of these regions confirms the results obtained: Cercospora oryzae was the dominant pathogenic species in the site of Gative, Docomey and Kpodji, Curvularia oryzae was the dominant pathogenic species in the Aizè site, Houeli Gaba, Zoungo, Magnaporthe grisaea corresponded to the dominant pathogenic species in the Zaloko site, Aizè, Docomey while Helminthosporium oryzae corresponded to the dominant pathogenic species in the site of Odja Igbagi, Awokpa, Kodé Ague. These differences are likely to be due to climate conditions and the different mode of cultivation between sites also in the use of seeds.

Keywords: Oryza sativa L., fungal pathogen, incidence, diagnosis, morphological identification

\section{Introduction}

La culture du riz (Oryza sativa L.) est une des principales filières vivrières du Bénin (Kodjo, 2012). Pour assurer la production du riz, les riziculteurs Béninois pratiquent principalement le système de riziculture des bas fonds (CCR-B, 2014). Malheureusement, ce système subit actuellement des contraintes physiques inéluctables. En effet, les surfaces rizicoles des bas fonds sont à présent en état de sursaturation à cause de la pression foncière liée à une croissance démographique galopante (Gbenou et Agossou, 2013). On assiste ainsi à une diminution et une dégradation de leur fertilité par l'épuisement du sol (CCR-B, 2014).

Par ailleurs, ces terres inondées sont victimes des dégradations de l'environnement qui engendrent des phénomènes d'érosion diminuant davantage les surfaces cultivables (CCR-B, 2014). Le report de la culture du riz sur les collines par le système de culture pluvial constitue une possibilité d'extension des surfaces afin de subvenir aux demandes de la consommation croissante. Mais l'aboutissement de cette alternative passe, entre autre, par la capacité du système à s'adapter au milieu et à lutter contre ses ennemis naturels, notamment les maladies et les ravageurs (Adegbola et Sigbo, 2003).

Le diagnostic agronomique vise à identifier les différentes maladies fongiques présentes dans le système de riziculture de bas-fond et celui pluviale au Sud du Bénin. Cette étude contribue à l'amélioration de la production du riz, du système de culture du riz pluvial et de Bas-fond dans le Sud du Bénin, dont l'objectif est de réaliser un diagnostic de l'incidence de la cercosporiose et des autres maladies fongiques dans cette région.

La cercosporiose, ou tache brune étroite, est causée par un champignon dont l'agent responsable est Cercospora oryzae (= Sphaerulina 
oryzae), elle survient pratiquement partout où le riz pousse. Son développement ou sa gravité peut cependant varier d'un endroit à l'autre.

Dans les années 1930 et 1940, la cercosporiose était un problème grave pour les producteurs de riz aux États-Unis, en particulier dans l'Arkansas, en Alabama, Louisiane et Texas (Tullis 1937, Ryker 1947). Dans les années 2005 au Sénégal et dans les années 2015 au Burkina Faso la cercosporiose à causée de nombreuses pertes aux producteurs. Au Bénin aucun travail n'a été mené sur la cercosporiose dans le sens d'évaluer la distribution et les pertes que cette maladie peut causer.

\section{Matériel et Méthodes}

\section{Milieu d'étude, prospections et évaluation de l'incidence et de la sévérité} des maladies

Une première prospection a été effectuée pendant la saison 2017 , alors qu'une deuxième prospection a été réalisée pendant la saison 2018, dans 6 départements (Atlantique, Mono, Ouemé, Plateau, Zou, Couffo), dans 9 communes (Zè, Abomey-Calavi, Comé, Grand-Popo, Adjohoun, Pobè, Adja Ouéré, Dogbo, Ouinhi) et sur 12 sites (Awokpa, Docomey, Gative, Matekpo, Kodé-Ague, Gouti, Odja-Igbagi, Houeli-Gaba, Kpodji, Aizè, Zaloko, Zoungo) situées au Sud Bénin.

$\mathrm{Au}$ cours de ces prospections et sur chaque sites, les échantillons ont été collectés en sillonnent les rizières, à prélever les échantillons de feuilles de riz présentant les symptômes de la maladie fongique telles définies par la littérature. Chaque échantillon est constitué de cinquante (50) feuilles placées dans des sachets en plastique et scellé avec du parafilm. Un nombre total de sept cent (700) feuilles ont été collectées lors de la prospection dans les localités visitées.

Après triage, cent quarante (140) feuilles ont été retenus. La mise en incubation est faite au fur et à mesure que les échantillons sont apportés du terrain. La répartition des échantillons par site de collecte est donnée dans le tableau 1. Les prélèvements sont réalisés au hasard selon la technique d'échantillonnage en diagonale décrite par Matsushima (Matshushima, 1966).

Tableau 1 : Variétés, sites, année de collecte des feuilles de riz

\begin{tabular}{|l|l|l|l|l|}
\hline $\begin{array}{l}\text { Numéro } \\
\text { d'échantillons }\end{array}$ & Variétés & Site de collecte & $\begin{array}{l}\text { Année de } \\
\text { collecte }\end{array}$ & $\begin{array}{l}\text { Nombre de feuilles } \\
\text { collectées }\end{array}$ \\
\hline $\mathbf{1}$ & IR841 & Zaloko & SH 2018 & 50 \\
\hline $\mathbf{2}$ & IR841 & Awokpa & SH2018 & 50 \\
\hline $\mathbf{3}$ & IR841 & Zoungo & SH2018 & 50 \\
\hline $\mathbf{4}$ & IR841 & Gouti & SH2018 & 50 \\
\hline $\mathbf{5}$ & IR841 & Aizé & SH2018 & 50 \\
\hline $\mathbf{6}$ & IR841 & Gocomey & SH2018 & 50 \\
\hline $\mathbf{7}$ & IR841 & Gative & SH2018 & 50 \\
\hline
\end{tabular}




\begin{tabular}{|l|l|l|l|l|}
\hline $\mathbf{8}$ & IR841 & Matekpo & SH2018 & 50 \\
\hline $\mathbf{9}$ & IR841 & Kodé Ague & SH2018 & 50 \\
\hline $\mathbf{1 0}$ & IR841 & Odja-Igbagui & SH2017 & 50 \\
\hline $\mathbf{1 1}$ & IR841 & Odja-Igbagui & SH2018 & 50 \\
\hline $\mathbf{1 2}$ & IR841 & Houéli-Gaba & SH2017 & 50 \\
\hline $\mathbf{1 3}$ & IR841 & Houéli-Gaba & SH2018 & 50 \\
\hline $\mathbf{1 4}$ & IR841 & Kpodji & SH2018 & 50 \\
\hline \multicolumn{7}{|l}{ SH : Saison humide } \\
\hline
\end{tabular}

L'évaluation de l'incidence des maladies a été effectuée sur 20 plants choisis au hasard sur chaque site suivant les diagonales. Le taux d'incidence des maladies a été estimé à partir du rapport entre le nombre de feuilles malades et le nombre total de feuilles inspectés pour chaque maladie (Cooke, 2006). Pour l'évaluation de la sévérité moyenne des maladies, une deuxième prospection a été organisée après avoir évalué les incidences des maladies dans les différents sites après la première prospection. Les sites de Gative, Docomey, Kpodji et Matekpo dans lesquelles les maladies présentaient les incidences les plus élevées ont été alors choisies. Par souci de représentativité de l'échantillon, l'évaluation a été faite sur les côtés Est, Ouest, Nord et Sud de chaque site. Sur chacun de ces côtés, un quadrant de $1 \mathrm{~m}^{2}$ a été délimité (Shomari et Kennedy, 1999), puis au niveau de chaque quadrant, le pourcentage de feuilles infectées de chaque maladie a été déterminé par comptage et rapporté au nombre total de feuilles dans le cadrant. Le pourcentage moyen de feuilles infectées par plant a été alors calculé et représente la sévérité moyenne de la maladie sur ce plant.

La sévérité de la cercosporiose a été évaluée en suivant une échelle visuelle de 1 à 9 (Moral et Trapero, 2009) où : $1=$ pas de symptôme, $2=1$ $4 \% ; 3=5-9 \% ; 4=10-19 \% ; 5=20-29 \% ; 6=30-44 \% ; 7=45-59 \% ; 8=$ $60-75 \% ; 9=>75 \%$ de pourcentage moyen de lésions par feuille infectée.

L'indice de sévérité de la maladie sur le site a été déterminé à l'aide de l'équation suivante $(\mathrm{Kranz}, 1988): \mathrm{Is}=\frac{\sum \quad(x i \times n i)}{N . Z} \times 100$, avec $:$ Is $=$ Indice de sévérité de la maladie sur le site, $x i=$ Sévérité $i$ de la maladie sur le plant, $n i$ $=$ Nombre de plants de sévérité $i, \mathrm{~N}=$ Nombre total de plants observés et $\mathrm{Z}=$ Echelle de sévérité la plus élevée, soit 9.

\section{Identification des pathogènes}

L'identification des agents pathogènes responsables des maladies du riz a été faite sur la base des symptômes observés au champ, et confirmée par des observations microscopiques et des isolements sur milieux artificiels de culture en utilisant les guides d'identification (Barnett et Hunter, 1987; Domsch et al., 1980) et en appliquant le postulat de koch. 
Les feuilles sont lavées soigneusement et abondamment à l'eau courante. Découpées par la suite en de petits fragments $2,5 \mathrm{~cm}$ présentant des symptômes (Estrada et Ou, 1978). Les fragments de feuilles sont désinfectées pendant quelques secondes dans de Hypochlorite de sodium à $2 \%$, ensuite rincées à l'eau distillée stérilisée pendant 30 secondes par trois fois (Hode, 2014), pour fin d'éliminer les microflores exogènes, et sont déposées sur papier filtre stérile (watmann) pour séchage pendant 30 secondes sous la hôte.

5 fragments de feuilles découpées seront déposées sur les milieux de culture PDA (Potato, Dextrose, Agar), Farine de riz et Agar-Agar préalablement coulés dans des boites de pétri. Certaines feuilles sont déposées sur du papier filtre imbibé d'eau distillée (méthode du papier buvard modifié) dans les boite de pétri (Gnancadja, 2005 ). Les boites sont mises en incubation pendant 3 à 4 jours pour les boites contenant les milieux de culture et 7 jours pour les boites avec le papier imbibé d'eau distillée le tous sous une température comprise entre 25 et $30^{\circ} \mathrm{C}$ afin d'induire la sporulation et la formation de conidies spécifiques aux agents pathogènes fongiques impliqués (Sy et Sere, 1996).

Certaines boites sont alternées pendant $12 \mathrm{~h}$ à la lumière et $12 \mathrm{~h}$ à obscurité.

\section{Analyses statistiques}

Les données recueillies sur le terrain ont été analysées avec le logiciel SPSS 16.0. Le test de Student-Newman-Keuls au seuil de 5\% a été utilisé pour la séparation des moyennes d'incidence et de sévérité des maladies.

\section{Resultats}

\section{Inventaire des pathogènes fongiques du riz}

Six pathogènes fongiques (Cercospora oryzae, Magnaporthe grisaea, Helminthosporium oryzae, Curvularia lunata, Nigrospora oryzae, Aspergilus) ont été identifiés sur l'ensemble des sites prospectés, dont les incidences moyennes les plus élevées ont été relevées pour cercosporiose $(50,98 \%)$, la pyriculariose $(35,24 \%)$ et la curvulariose $(32,96 \%)$. Ces trois maladies ont été considérées comme les plus importantes pour l'ensemble des sites comparativement à l'incidence moyenne de l'helminthosporiose qui était la plus faible $(2,18 \%)$. Les symptômes de la cercosporiose (Cercospora oryzae) se réduisent à plusieurs taches effilées de couleur brune, de forme rectiligne parallèle à la nervure principale sur la face supérieure des feuilles (Photo.1), tandis que ceux de la pyriculariose sont perceptibles sur les feuilles et sur les tiges. Les symptômes apparaissent sous forme de petits points bruns grisâtres qui deviennent rapidement des lésions typiques, brunes à centre grisâtre ou blanchâtre. Quant-à la curvulariose (Curvularia lunata), elle provoque 
plusieurs taches brun-sombres, circulaires ou ovales observées sur la face supérieure des feuilles.

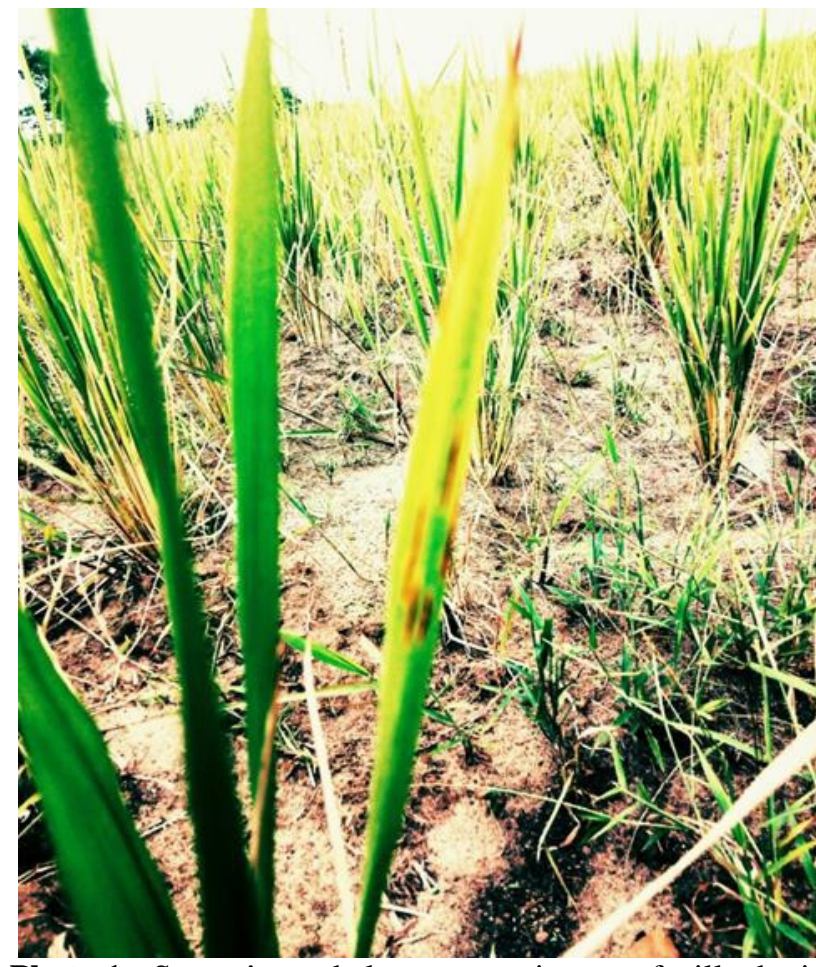

Photo 1 : Symptômes de la cercosporiose sur feuille de riz Source : Soura, 2018

\section{Identification Morphologique de Cercospora oryzae}

Cercospora oryzae a été isolée sur les feuilles de riz dans les différents sites prospectés. Après la mise en culture et l'isolement nous avons identifié les spores de Cercospora oryzae (photo 2) septés entre 4 à 5 lobes avec une extrémité effilée à l'autre extrémité arrondie. Les colonies sur milieu de culture sont blanc, cotonneux (photo 3) avec des mycéliums cloisonnés. 

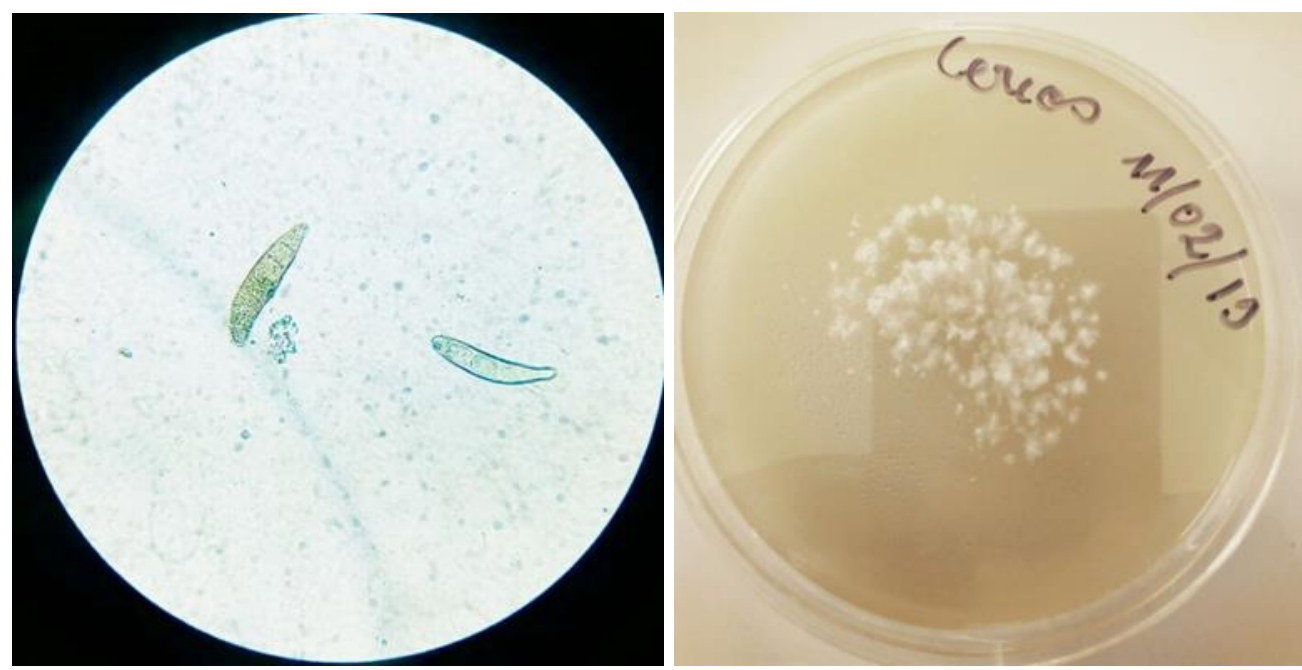

Photo 2 : Spores de Cercospora oryzae Source : Soura, 2018

Photo 3 : Cercospora oryzae sur milieu de culture Source : Soura, 2018

\section{Distribution des principales maladies du riz dans les zones de production}

L'incidence de la Cercosporiose, la pyriculariose, la curvulariose et de l'heminthosporiose considérées comme les principales maladies du riz inventoriées dans les rizières installées dans les 9 communes productrices de riz au Bénin était variable suivant les zones de production (Tableau 2). La Cercosporiose causée par Cercospora oryzae est d'une grande importance dans 6 sites sur les 12 sites prospectées et l'incidence a atteint 90\%, 79,2\% et 73,3\% respectivement sur les sites de Gative, Docomey et Kpodji. Par contre, la pyriculariose causée par Magnaporthe grisaea était beaucoup plus importante sur les sites de Zaloko (90,9\%) et de Aizè (75\%). La curvulariose causée par Curvularia lunata, quant à elle, était surtout présente dans les sites de Aizè (75\%), Houeli Gaba (51,1\%) et Zoungo (47,8\%) alors que l'helminthosporiose causée par Helminthosporium oryzae ne se retrouvait qu'à Odja-Igbagi, Kodé Ague, Gouti et Awokpa, avec de très faibles incidences (Tableau 1).

Tableau 2 : Incidences moyennes (\%) des maladies du riz par commune productrice au Sud du Bénin

$\begin{array}{llllll}\text { Communes } & \text { Pyriculariose } & \text { Cercosporiose } & \text { Curvulariose } & \begin{array}{l}\text { Helminthos } \\ \text { poriose }\end{array} & \text { Moyenne } \\ \text { Odja-Igbagi } & 29,2 \pm 4,4 \mathrm{cAB} & 16,0 \pm 3,6 \mathrm{abA} & 26,4 \pm 4,3 \mathrm{bcA} & 9,4 \pm 2,9 \mathrm{aA} & \mathbf{2 0 , 3} \pm \mathbf{2 , 0} \\ \text { Awokpa } & 21,7 \pm 5,0 \mathrm{aAB} & 50,7 \pm 6,1 \mathrm{bAB} & 18,8 \pm 4,7 \mathrm{aA} & 7,6 \pm 3,1 \mathrm{aA} & \mathbf{2 4 , 6} \pm \mathbf{2 , 6} \\ \text { Kodé Ague } & 8,8 \pm 3,8 \mathrm{aA} & 49,1 \pm 6,7 \mathrm{bAB} & 35,1 \pm 6,4 \mathrm{bA} & 7,0 \pm 3,4 \mathrm{aA} & \mathbf{2 5 , 0} \pm \mathbf{2 , 9} \\ \text { Zaloko } & 90,9 \pm 9,1 \mathrm{bD} & 27,3 \pm 14,1 \mathrm{aA} & 27,3 \pm 14,1 \mathrm{aA} & 0 \mathrm{aA} & \mathbf{3 6 , 4} \pm \mathbf{7 , 3} \\ \text { Aizè } & 75,0 \pm 13,1 \mathrm{bCD} & 25,0 \pm 13,1 \mathrm{aA} & 75,0 \pm 13,1 \mathrm{bB} & 0 \mathrm{aA} & \mathbf{4 3 , 8} \pm \mathbf{7 , 2} \\ \text { Houeli Gaba } & 12,8 \pm 4,9 \mathrm{aA} & 51,1 \pm 7,4 \mathrm{bAB} & 51,1 \pm 7,4 \mathrm{bAB} & 0 \mathrm{aA} & \mathbf{2 8 , 7} \pm \mathbf{3 , 3} \\ \text { Zoungo } & 17,4 \pm 5,7 \mathrm{bA} & 50,0 \pm 7,5 \mathrm{cAB} & 47,8 \pm 7,5 \mathrm{cAB} & 0 \mathrm{aA} & \mathbf{2 8 , 8} \pm \mathbf{3 , 3} \\ \text { Gouti } & 11,4 \pm 3,6 \mathrm{aA} & 45,6 \pm 5,6 \mathrm{cAB} & 26,6 \pm 5,0 \mathrm{bA} & 2,5 \pm 1,8 \mathrm{aA} & \mathbf{2 1 , 5} \pm \mathbf{2 , 3} \\ \text { Docomey } & 54,2 \pm 10,4 \mathrm{cBC} & 79,2 \pm 8,5 \mathrm{~dB} & 25,0 \pm 9,0 \mathrm{bA} & 0 \mathrm{aA} & \mathbf{3 9 , 6} \pm \mathbf{5 , 0}\end{array}$




$\begin{array}{llllll}\text { Matekpo } & 18,2 \pm 12,2 \mathrm{aA} & 54,5 \pm 15,8 \mathrm{bAB} & 9,1 \pm 9,1 \mathrm{aA} & 0 \mathrm{aA} & \mathbf{2 0 , 5} \pm \mathbf{6 , 2} \\ \text { Kpodji } & 53,3 \pm 13,3 \mathrm{bBC} & 73,3 \pm 11,8 \mathrm{bB} & 13,3 \pm 9,1 \mathrm{aA} & 0 \mathrm{aA} & \mathbf{3 5 , 0} \pm \mathbf{6 , 2} \\ \text { Gative } & 30,0 \pm 15,3 \mathrm{aAB} & 90,0 \pm 10,0 \mathrm{bB} & 40,0 \pm 16,3 \mathrm{aAB} & 0 \mathrm{aA} & \mathbf{4 0 , 0} \pm \mathbf{7 , 8} \\ \text { Moyenne } & \mathbf{3 5 , 2 4} \pm \mathbf{2 , 5} & \mathbf{5 0 , 9 8} \pm \mathbf{2 , 9 4} & \mathbf{3 2 , 9 6} \pm \mathbf{2 , 8 4} & \mathbf{2 , 1 8} \pm \mathbf{1 , 2 7} & \mathbf{3 0 , 3} \pm \mathbf{2 , 4}\end{array}$

Les valeurs moyennes suivies des mêmes lettres minuscules dans les lignes et celles suivies des mêmes lettres majuscules dans les colonnes ne sont pas significativement différentes au seuil de 5\% selon le test de Student-Newman-Keuls.

Concernant le niveau de sévérité des maladies, la Cercosporiose a présenté la sévérité moyenne la plus élevée $(18,19 \%)$ tandis que la curvulariose et la Pyriculariose avaient des sévérités moyennes respectives de $5,14 \%$ et $5,95 \%$ (Tableau 3). Pour la cercosporiose, les plus fortes valeurs ont été enregistrées dans les sites de Gative (25,3\%), Docomey (21,41\%), Kpodji (20,10\%) et Matekpo (19,70\%), tandis que pour la curvulariose, les sévérités les plus élevées ont été observées à Kpodji (13,73\%) et Matekpo (9,64\%). Quant à la Pyriculariose, les valeurs de sévérité enregistrées s'avoisinaient dans toutes les communes (Tableau 3).

Tableau 3 : Sévérités moyennes des maladies du riz par sites productrice au Bénin

$\begin{array}{lllll}\text { Communes } & \text { Curvulariose } & \text { Pyriculariose } & \text { Cercosporiose } & \text { Moyenne } \\ \text { Docomey } & 0,93 \pm 0,31 \mathrm{aA} & 7,25 \pm 1,27 \mathrm{aA} & 21,41 \pm 4,78 \mathrm{bB} & 9,9 \pm 1,8 \\ \text { Aizè } & 0,86 \pm 0,21 \mathrm{aA} & 3,50 \pm 0,47 \mathrm{aAB} & 4,44 \pm 1,65 \mathrm{aB} & 2,9 \pm 0,6 \\ \text { Gative } & 0,54 \pm 0,14 \mathrm{aA} & 5,92 \pm 1,44 \mathrm{aA} & 25,30 \pm 4,87 \mathrm{bB} & 10,6 \pm 1,9 \\ \text { Kpodji } & 13,73 \pm 4,28 \mathrm{bAB} & 5,85 \pm 0,88 \mathrm{aA} & 20,10 \pm 5,23 \mathrm{bB} & 13,2 \pm 2,3 \\ \text { Matekpo } & 9,64 \pm 3,44 \mathrm{bA} & 7,21 \pm 1,39 \mathrm{aA} & 19,70 \pm 4,27 \mathrm{bB} & 12,2 \pm 2,0 \\ \text { Moyenne } & \mathbf{5 , 1 4} \pm \mathbf{1 , 1 5} & \mathbf{5 , 9 5} \pm \mathbf{0 , 5 2} & \mathbf{1 8 , 1 9} \pm \mathbf{1 , 9 9} & \mathbf{9 , 8} \pm \mathbf{1 , 8}\end{array}$

Les valeurs moyennes suivies des mêmes lettres majuscules dans les lignes et celles suivies des mêmes lettres minuscules dans les colonnes ne sont pas significativement différentes au seuil de 5\% selon le test de Student-Newman-Keuls.

L'indice de sévérité de la cercosporiose calculé ne révélait pas de différence significative entre les sites. Cependant, les plus fortes valeurs ont été enregistrées dans les sites de Gative $(71,11 \%)$ et de Docomey $(66,67 \%)$ comme ce fut le cas avec l'incidence de la même maladie (Figure 1). 
Figure 1 : Indice de sévérité de la cercosporiose dans quelques sites productrices de riz au Sud Bénin

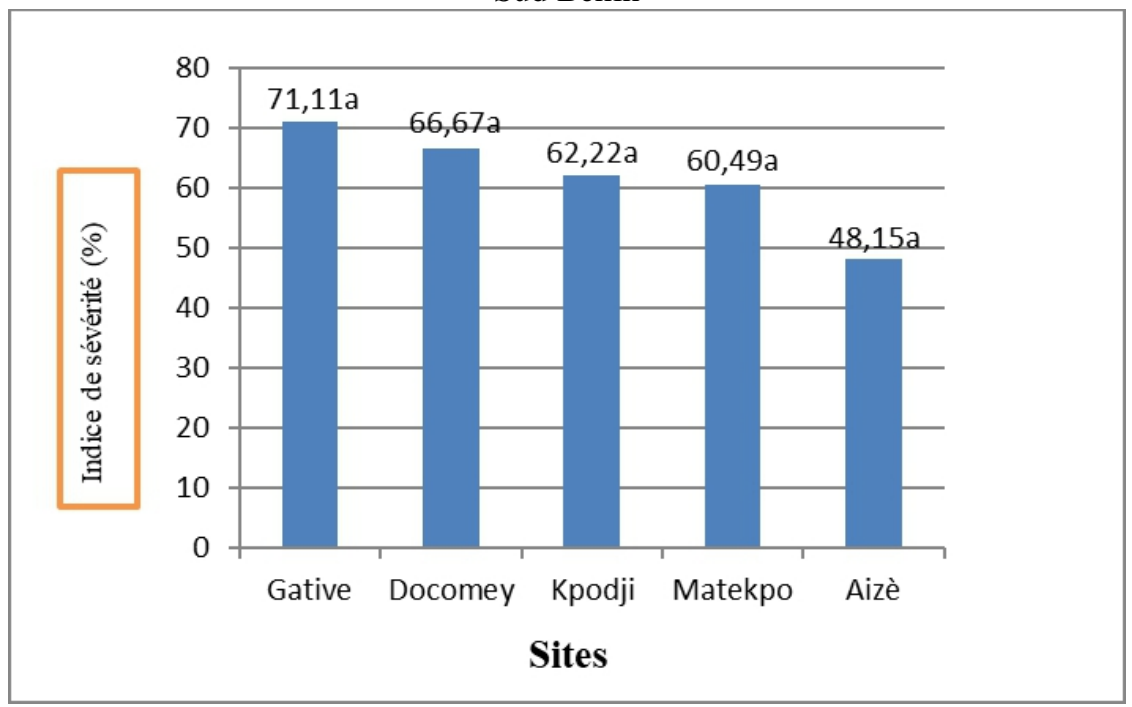

Les valeurs suivies d'une même lettre au-dessus des histogrammes ne sont pas significativement différentes au seuil de 5\% selon le test de Student-Newman-Keuls.

\section{Discussion}

Plusieurs maladies telles que la cercosporiose, la pyriculariose, la curvulariose et l'helminthosporiose sont identifiées sur le riz au Sud Bénin au cours de l'étude. Ces mêmes maladies avaient également été signalées sur le riz au Bénin (Hode, 2014), au Burkina Faso (Ouedraogo, 2008), au Maroc (Gnancadja, 2005). De toutes ces maladies, la cercosporiose, la pyriculariose et la curvulariose sont celles qui sont présentes dans toute l'aire de production de riz au Sud Bénin. Par conséquent, elles peuvent être considérées comme les principales maladies du riz au Sud Bénin, au regard de leur incidence et de leur sévérité.

Par ailleurs, l'étude nous permet de constater que l'heminthosporiose qui constitue une maladie majeure au Maroc n'a pratiquement pas d'importance au Bénin (Gnancadja, 2005 ; Hode, 2014). Toutefois, les conditions environnementales sont déterminantes dans l'étiologie de cette maladie et il convient de maintenir un état de veille dans un contexte de changement climatique et de beaucoup de légèreté dans l'application de la règlementation relative aux échanges transfrontaliers de matériel végétal.

Aussi, la distribution des principales maladies est variable d'un site à une autre. Ainsi, pendant que la cercosporiose est une préoccupation majeure dans les sites de Gative, Docomey, de Kpodji et de Matekpo c'est plutôt la curvulariose qui requiert un suivi particulier dans les sites de Aizè, Houeli Gaba, Zoungo. Quant à la pyriculariose, elle est beaucoup plus importante dans les sites de Zaloko, Aizè, Docomey. Des études épidémiologiques et de 
caractérisations morphologiques et moléculaires des souches de Cercospora présentes sur le riz au Sud Bénin sont nécessaires pour appréhender l'importance de la maladie et anticiper sur les méthodes appropriées de lutte.

En effet, plusieurs espèces et souches d'une même espèce de Cercospora responsables de dégâts importants sur le riz ont été identifiées en Louisane (Donald, et al., 2014) en Philippine (Estrada et Ou., 1978), Tullis, (1937) au Texas.

La présence de tous ces agents pathogènes sur le riz au Sud Bénin peut justifier en partie la chute des rendements dans le pays depuis quelques années.

Toutefois, la présente étude n'a pas pu apporter des informations sur l'impact réel de chacune des principales maladies identifiées sur le rendement qualitatif et quantitatif en graines au Sud Bénin. Il est alors nécessaire de poursuivre les travaux afin de préciser les maladies qui méritent une attention particulière dans l'élaboration des mesures de lutte dans chaque site de production du riz au Sud Bénin.

\section{Conclusion}

Notre étude révèle une complexité de maladie fongique présente sur les sites étudiés. La variété de riz IR841 supposée être résistante aux maladies est attaquée par plusieurs pathogènes fongiques notamment la cercosporiose causée par Cercospora oryzae en conditions de pression naturelle de maladie au champ. Les résultats obtenus au cours de notre étude font ressortir un impact négatif de la cercosporiose sur la productivité du riz. Il est souhaitable de poursuivre les investigations sur la pathologie afin de déterminer le pourcentage de perte réel causée par ce pathogène au champ. Aussi étudier la diversité au sein des populations et le niveau réel de pression parasitaire de Cercospora oryzae.

\section{References:}

1. Adegbola, Y. P., Singbo, A., 2005. Impact de 1'importation du riz sur la compétitivité et la rentabilité de la production nationale au Bénin. Communication scientifique. PAPA/INRAB $13 \mathrm{p}$.

2. Barnett, H. L., Hunter, B. B., 1987. Illustrated genera of imperfecti fungi. 4th edition, MacMilan, New York, USA.

3. CCR-B., 2014. Rapport de l'étude des lieux de la filière riz au Bénin en 2014. Secrétariat Permanent ; 75p.

4. Codjio, P. V., 2012. Caractérisation des bas-fonds en exploitation dans le sous-bassin versant de Zagbo (commune de Za-Kpota) : cas du basfond de Dogbanlin. FLASH, Mémoire, 79p.

5. Cooke, B. M., 2006. Disease assessment and yield loss: 43-80. In: Cooke, B.M., Jones, D.G., Kaye, B. (eds), The epidemiology of plant diseases. Springer, Netherlands, $\mathrm{p}$. 
6. Domsch, K. H., Gams, W., Anderson, T. H., 1980. Compendium of soil fungi. Vol. 1. Academic Press, London, U.K. 400-520pp.

7. Donal, G., Crowley, L.A and Clayton Hollier., 2004. Rice Cercospora disease complex management. LSU AgCenter, Department of Plant Pathology and Crop Physiology, Baton Rouge LA, 17p.

8. Estrada, B. A., et Ou, S. H., 1978. Méthodes de sélection des variétés de riz pour leur résistance a la cercosporiose du riz. IRRI Research paper series P0. Box 933. Manila, Philippines, 9p.

9. Gbenou, P., Agossou, N., 2013. Evaluation participative du Système de Riziculture Intensive dans la basse vallée de 1'Ouémé au Bénin. Thèse de doctorat en agronomie, Bibliothèque FLASH , FLASH/UAC . 214p.

10. Gnancadja L. S. A., 2005. Effet de certaines espèces fongiques sur le développement de la ternissue des grains de riz. Rev Inst Agr. Vét. Hassan II, Vol 5. 9p.

11. Hode G. Y., 2014. Etude de la flore pathogène et effet des agents pathogènes sur la qualité des grains de quelques variétés de riz (Oryza sativa L.) paddy cultivées au Bénin. Mémoire de Master en Biologie Végétale Appliquée Université d'Abomey-Calavi, 70p.

12. Kranz, J., 1988. Measuring plant disease: 35-50. In: Kranz, J., Rotem, J. (eds), Experimental techniques in plant disease epidemiology. Springer, Berlin, 4p.

13. Matshushima, S., 1966. Crop Science in rice: theory of yiels determination and its application. Tokyo: Fuji. Co. 365 p.

14. Moral, J., Trapero, A., 2009. Assessing the susceptibility of olive cultivars to anthracnose caused by Colletotrichum acutatum. Plant Disease, 93, 1028-1036.

15. Ouédraogo, I., 2008. Incidence de l'helminthosporiose du riz au Burkina Faso et caractérisation des populations de l'agent pathogène [Bipolaris oryzae (Breda de Haan) Shoemaker]. Thèse de doctorat Université de Ouagadougou, Science Biologique Appliquée option phytopathologie. $150 \mathrm{p}$.

16. Ryker, T. C., 1947. New pathogenic races of Cercospora oryzae affecting rice. Abs. in Phytopathology 37: 19-20.

17. Shomari, S. H., Kennedy, R., 1999. Survival of Oidium anacardii on cashew (Anacardium occidentale) in southern Tanzania. Plant Pathology 48, 505-513.

18. Sy, A. A., Séré, Y., 1996. Manuel de formation en pathologie du riz, ADRAO. Bouaké, Côte d'Ivoire. 94 p.

19. Tullis, E. C., 1937. Cercospora oryzae on rice in the United States. Phytopathology 27:1007-1008. 